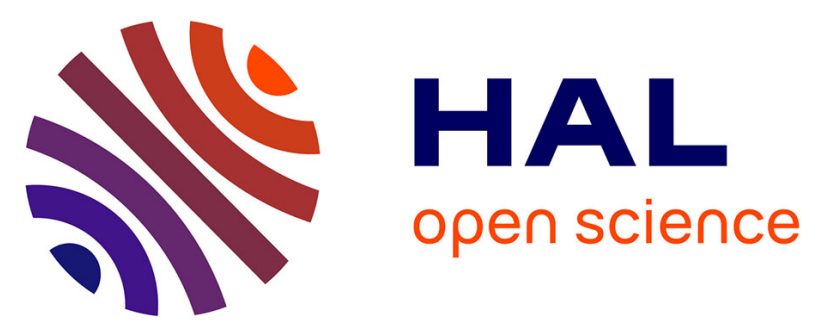

\title{
Quantitative Magnetic Resonance Imaging Assessment of the Quadriceps Changes during an Extreme Mountain Ultramarathon
}

Hoai-Thu Nguyen, Thomas Grenier, Benjamin Leporq, Caroline Le Goff, Benjamin Gilles, Sylvain Grange, Rémi Grange, Grégoire Millet, Olivier Beuf, Pierre Croisille, et al.

\section{To cite this version:}

Hoai-Thu Nguyen, Thomas Grenier, Benjamin Leporq, Caroline Le Goff, Benjamin Gilles, et al.. Quantitative Magnetic Resonance Imaging Assessment of the Quadriceps Changes during an Extreme Mountain Ultramarathon. Medicine and Science in Sports and Exercise, 2021, 53 (4), pp.869-881. 10.1249/mss.0000000000002535 . hal-02998029

\section{HAL Id: hal-02998029 https://hal.science/hal-02998029}

Submitted on 3 Jan 2021

HAL is a multi-disciplinary open access archive for the deposit and dissemination of scientific research documents, whether they are published or not. The documents may come from teaching and research institutions in France or abroad, or from public or private research centers.
L'archive ouverte pluridisciplinaire HAL, est destinée au dépôt et à la diffusion de documents scientifiques de niveau recherche, publiés ou non, émanant des établissements d'enseignement et de recherche français ou étrangers, des laboratoires publics ou privés. 


\section{Quantitative Magnetic Resonance Imaging 2 assessment of the Quadriceps changes during an 3 extreme mountain ultramarathon}

4

5

Email and postal address of corresponding author:

20 magalie.viallon@creatis.insa-lyon.fr

21 Service de radiologie

22 CHU de Saint Etienne

23 Avenue Albert Raimond

$24 \quad 42055$ Saint Etienne Cedex 2. 


\section{ABSTRACT}

\section{Introduction/Purpose}

27 Extreme ultra-endurance races are growing in popularity but their effects on skeletal 28 muscles remain mostly unexplored. This longitudinal study explores physiological 29 changes in mountain ultramarathon (MUM) athletes' quadriceps using quantitative 30 magnetic resonance imaging (qMRI) coupled with serological biomarkers.

31 The study aimed to monitor the longitudinal effect of the race and recovery, and to 32 identify local inflammatory and metabolic muscle responses by codetection of biological 33 markers.

\section{Methods}

35 An automatic image processing framework was designed to extract imaging-based 36 biomarkers from qMRI acquisitions of the upper legs of 20 finishers at three time points.

37 The longitudinal impact of the race was demonstrated by analyzing the image markers 38 with dedicated biostatistical analysis.

\section{Results}

40 Our framework allows a reliable calculation of statistical data not only inside the whole 41 quadriceps volume but also within each individual muscle heads. Local changes in MRI 42 parameters extracted from quantitative maps were described and found significantly 43 correlated with principal serological biomarkers of interest. A decrease in the PDFF after 44 the race and a stable paramagnetic susceptibility value were found. Pairwise post hoc tests suggested that the recovery process differs among the muscle heads. 


\section{Conclusion}

47 This longitudinal study conducted during a prolonged and extreme mechanical stress, 48 showed that quantitative MRI-based markers of inflammation and metabolic response 49 can detect local changes related to the prolonged exercise, with differentiated 50 involvement of each head of the quadriceps muscle as expected in such eccentric load.

51 Consistent and efficient extraction of the local biomarkers enables to highlight 52 interplay/interactions between blood and MRI biomarkers. This work indeed proposes 53 an automatized analytic framework to tackle the time consuming and mentally 54 exhausting segmentation task of muscle heads in large multi-time-points cohorts. 


\section{KEYWORDS}

56 Musculoskeletal; statistical analysis; image processing; functional variation 


\section{INTRODUCTION}

58 Mountain ultra-endurance running consists of running/walking outdoors over a distance longer than the traditional marathon on mountain trails with significant upward and downward slopes. This sport has become increasingly popular over the last decade.

Because of the inherent challenge for the body, the mountain ultramarathon (MUM) is

62 considered an outstanding model to investigate the adaptive responses to extreme load and mechanical stress(1) in skeletal muscles. Studies have been carried out to explore the impact of these prolonged cardiovascular and muscle efforts, demonstrating, for instance, multiple visceral changes with inflammatory effects(2-4) or an impairment of neuromuscular function(5). Among all muscles, the quadriceps are particularly targeted because extensive downhill running causes repeated eccentric contractions and high mechanical stress, ultimately leading to muscle damage $(6,7)$.

Magnetic Resonance Imaging (MRI) is a reliable, non-irradiating and non-invasive imaging method for tissue characterization and quantitative assessment of tissue integrity through its magnetic properties. Indeed T2 MR relaxation times, and its corresponding rates R2 (=1/T2) denote the characteristic time constants of the recovery back toward equilibrium of the $x y$ (transverse) components of the magnetization. After being disturbed, the MR signal source, i.e. nuclear magnetization, does not spontaneously recover very fast, but relies almost entirely on interactions of hydrogen nuclei with the surrounding material to re-equilibrate(8). Because T2 is sensitive to low-frequency components, in tenth of ms, water molecules diffuse and sample many different environments on the cellular level within the timescale of relaxation. In many (although by no means all) situations, very rapid exchange may occur between bulk water and bound and interfacial water in biological systems. This is believed to be the origin for many $\mathrm{T} 2$ changes in various pathologies, such as edematous changes following insults to tissue. Changes in tissue water and protein content have been shown to affect relaxation. The measure of 
82 average water proton relaxation rate will depend on how effectively, and at what rate, these

83 effects are spread through the rest of the water population. Moreover, the existence of water in

84 separate compartments that are only slowly exchanging gives rise to more complex

85 behavior(9). Many studies witness that edematous areas in the muscle appear bright in T2-

86 weighted images because the T2 relaxation time becomes longer, and T2 maps show elevated

87 quantitative values in the matching area(10,11). T2 maps have, therefore, been used to monitor inflammation in muscle diseases, before and after medical interventions or treatment(12-14), or to predict return to play(15). T2* is the "effective" $\mathrm{T} 2$ resulting from inhomogeneities in the main magnetic field or susceptibility-induced field distortions produced in the tissue, related to the presence of chemical or paramagnetic substances such as fat or hemorrhage(16). However, the first step in decoding the various effects of prolonged exercise stress on the body using qMRI markers is to develop systematic and comprehensive noninvasive exploration strategies to consistently extract the biomarkers reflecting the physiological consequences of such challenges.

96 From that perspective, magnetic resonance imaging (MRI) appears to be an unique imaging 97 modality for extracting anatomical and structural features of muscle tissue $(17,18)$. Recently, quantitative imaging methods such as chemical shift-encoded MRI(19,20) and MR relaxometry mapping $(21,22)$ have allowed users to understand chemical alterations noninvasively at the imaged pixel size.

101 Several postprocessing methods need to be sequentially applied to extract a quantitative index 102 from MRI data. These methods include the segmentation of muscle heads on multiple large 3D images, the extraction of image features in each area of interest, and statistical analysis. Each

104 of these steps is an area of research in its own right. Although various alternatives have been 105 proposed to target each of these challenges $(17,18)$, they are rarely applied to cohorts or 106 longitudinal studies. 
107 The aim of the study was to quantify the response of the quadriceps muscle to a MUM's 108 extreme loading conditions with quantitative MRI (qMRI) and to monitor the longitudinal

109 effect of race and recovery. To do so, we propose an automatized and integrated analysis

110 pipeline that enables the extraction of local quantitative imaging markers. Hence, the relation

111 of these new markers with standard blood biomarkers was investigated to provide further

112 insight into the local inflammatory and metabolic muscle responses.

\section{MATERIALS AND METHODS}

\section{Subjects and experimental study design}

116 We performed a longitudinal study on the athletes who participated in the "Tor des Géants"

117 MUM in 2014, including three MRI sessions carried out just before the start and, for those who

118 finished the race, immediately upon arrival and after 48 hours of recovery. Biological samples

119 were also drawn at four time points with an additional sample obtained at half-race. The 120 flowchart of the study is shown in Figure 1.

121 This study was approved by the local ethical committee (Aosta Valley, Azienda USL 101/946),

122 and the experimental plan was conducted in accordance with the Helsinki Declaration (2001).

123 Subjects were recruited through mailing and public announcements to registered runners by 124 race organizers. Exclusion criteria were smoking, substance abuse, regular intake of 125 medications, medical or psychiatric illness, and any contraindication to MRI (e.g., 126 claustrophobia, nonremovable metal devices) or abnormalities detected upon laboratory 127 screening. There were 50 experienced runners who volunteered and provided informed written 128 consent to participate in this study.

129 The Tor des Géants is a $330 \mathrm{~km}$ long ultradistance trail running, with considerable 130 positive/negative elevation changes $(+24,000 \mathrm{~m})$ in the Valley of Aosta (Italy). It is considered 
131 one of the most difficult mountain marathon races in the world since the ultra-endurance

132 activity is associated with high altitude exposure and sleep deprivation. The altitude along the

133 course ranges between 322 and 3,300 m, with 25 mountain passes over 2,000 m. The maximum

134 time allowed to complete the race is $150 \mathrm{~h}$, and in 2014 , the best performance was $71 \mathrm{~h} 49 \mathrm{~min}$

135 among 740 starters and 446 (60\%) finishers (http://www.tordesgeants.it/).

136 The present experimental design was thus a longitudinal study with repeated assessments at 137 four checkpoints:

138 - The first point (prerace: Pre) was at the start location. The data collection was performed within 4 days before the race and consisted of MRI acquisition and biological sampling.

- The second point was located halfway through the race (middle: Mid). Only biological sampling was performed.

- The third one was at the arrival of the race (arrival: Post): athletes who finished the race were transported by car to the laboratory and were evaluated (MRI and biological sampling) within $1 \mathrm{~h}$ after finishing the race.

- The last point (recovery: Post+3) was 48-72 h after arrival time. Both MRI and biological sampling were acquired.

\section{MRI acquisitions}

149 At Pre, Post and Post +3 time points, MRI acquisitions were performed on site using a mobile 1501.5 T MR scanner system (MAGNETOM Avanto, Siemens Healthcare, Erlangen, Germany, 151 located within a truck from Alliance Medical, England). A standard coil configuration was 152 used: a flexible 4-channel array surface coil, combined with 4 elements of the spine coil 153 embedded in the bed, resulting in an 8-channel coil in total.

154 Three MRI acquisitions of the legs were sequentially performed: 
- A three-dimensional (3D) isotropic gradient dual-echo sequence was used. The coronal acquisition included the entire upper leg (from the tibial tuberosity to the anterior superior iliac spine). The reconstruction of the water and fat images from the acquired dual-echo data sets was performed inline (Syngo software, Siemens healthcare, Erlangen, Germany) using a Dixon approach enabling four 3D isotropic in-phase, outphase, fat and water coronal images to be calculated inline on the MR scanner, hereafter denoted water (W), fat (F), in-phase (IN) and out-phase (OUT) images, respectively.

A 3D spoiled gradient echo sequence (3D GRE) was used for simultaneous estimation of proton density fat fraction (PDFF), $2^{*}$, and internal magnetic susceptibility $(\chi)$ quantification. Eight echoes were acquired in the transverse plane with a flyback readout gradient (first echo: $1.58 \mathrm{~ms}$ and echo spacing: $2.52 \mathrm{~ms}$ ). TR and flip angle were adjusted to minimize the T1-related bias. Phase and magnitude images were systematically reconstructed. Prescription of localization was performed using anatomic images from an isotropic 3D gradient echo acquisition. For standardization purposes, the central partition in the $\mathrm{z}$-direction was planned at a $15 \mathrm{~cm}$ distance from the upper part of the patella using the sagittal multiplanar reconstruction of the first acquired 3D gradient isotropic sequence.

A 2D multiecho T2 weighted spin-echo sequence (meSE) with 16 echo times (TEs) ranging from 10 to $178 \mathrm{~ms}$, with its central slice was also planned at the same location as previously described.

175 Each individual sequence was individually optimized. Anatomical regions explored by each

177 Digital Content 1, MR imaging acquisition parameters.

\section{MR Image postprocessing}


PDFF, $\chi$, and T2* were calculated from 3D-GRE acquisition using the reconstruction method

181 described in (19-22) which encompassed two main steps: fat-water separation (provided

182 parametric T2* and PDFF maps) and magnetic susceptibility quantification. Meanwhile,

183 T2 parametric maps were calculated from the meSE sequences.

184 An automatic segmentation based on external-force-driven deformable registration was

185 performed on the T1-W images (23) to obtain the quadriceps heads of interest.

186 Briefly, dedicated in-house postprocessing pipelines were required 1) to simultaneously

187 compute the main parametric maps (fat fraction - PDFF, internal magnetic susceptibility $(\chi)$

188 and $\mathrm{T}_{2} *$ from the 3D GRE sequence, $\mathrm{T} 2$ parametric map from the meSE sequence) and 2) to

189 correct the results of the automatic muscle segmentation and to adjust their resolutions to

190 different image sequences (Figure 2). These steps allowed us to extract metrics from the four

191 parametric maps: PDFF, $\chi, \mathrm{T} 2 *$ and T2. All details about parametric map reconstruction and

192 muscle segmentation are provided in Supplemental Digital Contents 2, quantitative maps

193 computation, and 3, muscle segmentation procedure.

194

\section{Biological sampling and analysis}

196 Blood and urinary samples were collected at each of the four sessions (Pre, Mid, Post, Post+3)

197 within 10 min after arrival at each key point. Blood samples were drawn from an antecubital

198 vein into a dry, heparinized, or EDTA tube according to the analysis to be performed and

199 immediately centrifuged. Since it was not possible to carry out all the investigations on the

200 same day by point-of-care technologies, plasma and serum were frozen at $-80^{\circ} \mathrm{C}$ within $20 \mathrm{~min}$

201 after blood collection for later analysis of muscle injury markers and biochemical variables.

202 The hematology parameters (hemoglobin, red blood cells, white blood cells) were directly

203 analyzed by a pocH-100i ${ }^{\mathrm{TM}}$ automated hematology analyzer (Sysmex, Villepinte, France).

204 Cobas 8000 (RocheDiagnostics, Manheim, Germany) was used to perform serial 
205 determinations for C-reactive protein (CRP), urinary creatinine, creatinine, calcium, chlorine, 206 potassium, sodium, and cholesterol. The osmolality and urinary osmolality were measured on an Arkray Osmo Station OM-6050 (Menarini, Florence, Italy).

208 All blood biomarkers analyzed in this study are listed in Supplemental Digital Content 4, 209 complete list of 58 biological markers analyzed in the study.

\section{Statistical analysis}

212 Missing values in biological datasets were estimated (see Supplemental Digital Contents 5, 213 biological data preprocessing for missing data) to employ paired statistical tests and for 214 subsequent correlation analysis between MRI and biological markers. The significance level 215 was set at $\mathrm{P}<.05$ for all statistical tests.

\section{Longitudinal statistical analysis}

217 For the qMRI longitudinal data, we used a statistical analysis of repeated measures with 218 adaptation to the data normality, as the normal distribution could not be assumed. For each qMRI calculated index of each of the 9 muscle volumes (4 quadriceps muscle heads per leg

220 and the total quadriceps volume), we tested the normality of the data at 3 time points with the

221 Shapiro-Wilk test. For the global effect test, one-way ANOVA designed for repeated measures was conducted for all data normally distributed at all the time points; otherwise, the Friedman test was employed.

224 While performing ANOVA, the sphericity of the data was verified by using Mauchly's test. If 225 the sphericity assumption was violated, the Greenhouse-Geisser correction method was used 226 on the P-value of ANOVA. After the global effect test, a post hoc test was performed to 227 compare each pair of time points. The type of post hoc test depended on the normality of differences between two time points: dependent t-test for normally distributed differences and 
229 Wilcoxon signed rank test otherwise. The obtained P-values were adjusted with the Bonferroni 230 adjustment method for multiple comparisons.

231 A similar strategy was applied to the biological marker data with 58 variables at 4 time points.

232 Relative variations of a biomarker $X$ at each time point $\left(R V\left(X_{n}\right), n \in\{\right.$ Mid, Post, Post +3$\left.\}\right)$

233 compared to time point Pre were also computed with:

$$
R V\left(X_{n}\right)=\frac{\overline{X_{n}}-\overline{X_{\text {Pre }}}}{\overline{X_{\text {Pre }}}} \times 100 \%
$$

235 where $\overline{X_{n}}$ is the average value of biomarker $\mathrm{X}$ at time point $n$.

236 Associations between image markers and biological markers

237 The potential associations between image markers and biological markers were evaluated by 238 calculating the repeated measure correlation coefficient(24) between each image marker and 239 each biological marker. 


\section{RESULTS}

\section{MR markers: parametric mapping analysis}

243 Of the 50 athletes that were included in the study, 31 finishers completed the race $(62 \%)$ in an 244 average time of $124 \pm 13$ h. Among them, only those with quadriceps muscle MRI datasets 245 available at all the time points $(\mathrm{n}=24)$ and with quantitative maps free of artifacts at all three 246 time points were retained for final analysis $(n=20)$. Artifacts leading to data exclusions were 247 mostly motion-related. They were specifically due to uncontrolled motion of the legs typically 248 observed immediately after long races potentially combined with those related to sudden sleep 249 attacks during MR acquisitions. Demographic data and global qMRI metrics collected at each 250 time point are reported in Supplemental Digital Content 6, demographic data and qMRI metrics 251 of the subject population.

252 The first step was to explore differences among all quadriceps muscle heads. Figure 3 shows a matrix of the t-test results when comparing qMRI metrics ( $\chi$, PDFF, T2, T2*) between all

254 muscle heads at the three MR acquisition time points (Pre, Post, Post+3). When focusing only 255 on right/left differences of the corresponding muscle heads, $\chi$, T2* and PDFF showed similar tendency. At the same time, there was no significant difference between right and left muscle heads for $\chi$ and $\mathrm{T} 2 *$ at any time point, and PDFF exhibited a significant difference between 258 vastus lateralis (VL) heads only at a single time point (Post +3$)$. On the other hand, the T2 259 metric showed a different pattern with a significant difference between the rectus femoris (RF) 260 heads of the two legs at the three time points (Pre, Post, Post +3 ) and a significant difference 261 between the left and right vastus medialis (VM) at time point Pre. Different muscle heads also 262 had different qMRI values, especially the RF, which showed significant differences compared 263 to all the other muscle heads most of the time. These results highlight the need to consider 
individual muscle behavior separately and that pooling quadriceps muscle heads may result in

265 a loss of information.

266 When analyzing temporal changes on the repeated measures of qMRI metrics, we observed a 267 significant time effect (i.e., race effect) on T2* and T2 mean values. This is shown in Figures

2684 and 5 with typical source images (see also Supplemental Digital Content 6 - Demographic 269 data and qMRI metrics of the subject population, for summary values and Supplemental Digital 270 Content 7 - Specific longitudinal T2* analysis, for a local analysis of right VI in T2* for an 271 example subject). Both T2* and T2 were significantly longer at arrival for most of the muscle 272 heads. They significantly decreased after recovery for the VM and the vastus intermedius (VI) 273 while not returning to baseline values at that time of measurement.

274 PDFF and $\chi$ showed only a small time effect (Figure 6-7). Pooling all muscles, PDFF had a 275 tendency to decrease after the race $(2.88 \pm 0.53)$ compared to baseline $(3.16 \pm 0.45)$ while 276 increased to higher values than baseline $(3.17 \pm 0.46)$ after $48 \mathrm{~h}$ of recovery. When considering 277 each muscle, the time effect reached significance for both VI, right VM and left VL muscle 278 heads (Figure 6). Despite a similar trend as the PDFF, time-effect changes in $\chi$ did not reach 279 significance for most muscle heads, except for left VL and left VM (L).

280 When focusing on $\mathrm{T} 2 *$ and T2 findings, the vastus group exhibited stronger variations than the 281 RF muscle group. The VM and VI had larger changes than the VL or the average of all muscles. $282 \mathrm{~T} 2 *$ values appeared less sensitive to muscle changes than $\mathrm{T} 2$, as illustrated in Figure 3, but 283 most of the statistical tests were significant except for some muscle heads on both legs between 284 time points Post and Post+3.

\section{Biological marker analysis}

286 The longitudinal variations in biological markers throughout the race are shown in 287 Supplemental Digital Content 8, Values of urinary and blood biomarkers at 4 time points. As 
main blood biomarkers of muscle damage, serum creatine kinase (CK) and myoglobin levels peaked at $+6598 \%$ and $+4159 \%$ at Mid, while serum lactate dehydrogenase (LDH) levels peaked at $+240 \%$ at Post and were elevated at Mid at $+197 \%$ (all P-values of post hoc test

291 against Pre are less than 0.01, see Figure 8). For most of the biomarkers, the biomarker values 292 at Pre were significantly different from those at the other time points (Mid, Post, Post +3 ), while 293 the difference between Mid and Post was nonsignificant.

\section{Correlation between qMRI features and main blood biomarkers}

295 We presented our results with a heatmap produced within R software (Supplemental Digital 296 Content 9, Correlation between biological markers and image features extracted from 297 quadriceps muscles in each qMRI quantitative map). An interactive version of this heatmap 298 can be found online 299 (http://amp.pharm.mssm.edu/clustergrammer/viz_sim_mats/5bed953490fbf7585f568 300 0c2/Correlation_Mean.txt ) thanks to Clustergrammer(25). Significant correlations between 301 qMRI features and biological markers were found:

302 - The T2 mean extracted from the whole quadriceps showed a significant time effect at all time 303 points of the race and a relatively strong correlation with blood/urine biomarkers such as 304 myoglobin (0.69), CK (0.78) and LDH (0.72). At the level of individual muscles, the 305 correlation coefficient is higher for VI and VM muscles.

$306-\mathrm{T} 2 *$ exhibited similar results but with lower correlation coefficients: 0.54 for myoglobin, 0.57 307 for CK and 0.61 for LDH.

308 - Meanwhile, neither the mean global PDFF nor $\chi$ showed any significant correlation 309 coefficients with biological markers in this study. 
312 While ultraloading events such as MUMs are recognized to endanger the musculoskeletal

313 system, little is known about the exact consequences of the acute biomechanical burden on

314 musculoskeletal tissues. Although (26) recommends the use of MRI as the method of choice

315 for studying endurance-related damage, to date, there has been only one longitudinal

316 observational field cohort study, focusing primarily on changes in overall body composition

317 (27) or morphological tissue damage(28).

318 The challenge and novelty of this work are two-fold: 1) first, to provide a technological 319 approach specifically developed enabling the extraction of qMRI parameters for longitudinal 320 skeletal muscle studies; 2) then, to demonstrate that qMRI brings unique insights into the 321 response of quadriceps muscle exposed to prolonged mechanical stress of a $330 \mathrm{~km}$ ultra322 endurance event.

323 To do so, we built a standardizing and automatic analysis pipeline that segments, calculates, and extracts all qMRI markers in each quadriceps muscle's heads that can be robustly 325 propagated to the different follow-up time points, thus enabling longitudinal monitoring.

326 Beyond the scope of this work, our first contribution is to empower a comprehensive and 327 multiparametric quantification of MRI biomarkers in thigh skeletal muscle, including T2 and $328 \mathrm{~T} 2 *$ relaxation times, together with fat content (PDFF) and internal magnetic susceptibility $\chi$. 329 Sencondly, the analysis framework enabled analysis of each quadriceps muscle head over 330 different time points (see Supplemental Digital Content 6, Demographic data and qMRI 331 metrics of the subject population, and Figures 4-7).

332 Our main findings are that the cumulative biomechanical stress of a $330 \mathrm{~km}$ MUM onto 333 quadriceps muscles translated in our subjects to a clear inflammatory burden with an increase 334 in $\mathrm{T} 2$ and $\mathrm{T} 2 *$ relaxation times. On the other hand, muscular metabolic and energy storage 
335 status probed fat content (PDFF) remained relatively stable with a small decrease in PDFF, 336 contrasting with the high energy expenditure that mainly relies on lipids. Below is a detailed

337 discussion of each qMRI finding.

$338 T 2$ and $T 2 *$ changes: MR markers of the muscular inflammatory burden

339 T2 and T2* changes (Supplemental Digital Content 6, Demographic data and qMRI metrics of

340 the subject population, and Figures 4, 5) are an expression of the well-known sensitivity of 341 qMRI T2 and T2* relaxation time parameters to overall water content variations and water 342 redistribution between intracellular and extracellular space(18,22,29-32). Our findings 343 confirm previous studies that reported with other approaches a significant inflammatory 344 response after $\operatorname{MUM}(5,33)$, mainly related to repetitive eccentric muscle contractions resulting 345 in microscopic muscle damage and subsequent edema $(5,6,34)$. In our study, T2 and T2* 346 remained elevated even after a few days of recovery, suggesting that $48-72 \mathrm{~h}$ is not sufficient 347 for a full recovery in most subjects and confirming previous findings by Robach et al(35).

348 Relaxometry changes demonstrated at the muscular level were corroborated by a large increase 349 in skeletal myocyte injury-related biomarkers (CK, myoglobin and LDH) that was also 350 measured in our subjects. These blood biomarkers are known to witness microlesions and early 351 signs of cellular inflammatory processes preceding water redistribution from intracellular to 352 extracellular compartments and have also been shown to reflect the extent of cellular 353 damage (33,36). Among the other findings in the biological profiles, WBC activation, 354 particularly neutrophil activation, is known to trigger cellular and humoral inflammatory 355 responses(37) characterized by elevated CRP levels and the presence of leucocytes in the 356 extracellular and extravascular spaces in response to long-term exercise $(33,38)$. The intrinsic 357 strength of MR imaging relies on its capacity to precisely reveal the local changes, while the 358 evident correlation of quantified $\mathrm{T} 2$ and $\mathrm{T} 2 *$ parameters with biological markers most likely 
could reflect a difference in the temporal response of the compared biomarkers. If the tendency

360 are similar, the amplitude of the correlation could only be due to a difference in the temporal

361 occurrence of the inflammation signature at the blood and tissue level, hence also during the

362 tissue recovery process.

363 The development of peripheral and muscular edema together with a total body water increase

364 have been reported in the context of ultramarathon running(27), with a $6 \%$ increase in total

365 body water after a 1,200 km run over 17 consecutive days and an increase in total body water

366 associated with tissue edema(39). Our measures of the total quadriceps muscle volume

367 (excluding superficial tissues) illustrated the same trend but with a small increase in quadriceps

368 muscle volume $(+3.3 \%$ at the end of the race and $+3.4 \%$ after $48-72 \mathrm{~h}$ recovery relative to

369 baseline; see Supplemental Digital Content 6, Demographic data and qMRI metrics of the

370 subject population): number of voxels from the 3D GRE sequence, expressing muscle volume).

371 This observation suggests that among the potential mechanisms involved, the T2 and $\mathrm{T} 2 *$

372 changes are more likely related to water redistribution than to a net muscular tissue water

373 content increase.

374 An interesting finding is the observable difference in $\mathrm{T} 2$ and $\mathrm{T} 2 *$ response among the different 375 quadriceps muscle heads. Indeed, while the VI and VL exhibited both higher T2 and T2* 376 increases, the RF showed almost no variations. These findings confirm previous studies 377 exploring MUM and long-distance running adaptation strategies. Sloniger et al.(40) For instance, uphill running requires considerably greater activation of the vastus group and less activation of the RF than horizontal running(40). Ultrarunners are also known to modify their running pattern by increasing their stride frequency and by reducing the vertical oscillations to

381 minimize the load and the associated pain occurring in lower limbs mainly during the eccentric 382 phases(41). Impact forces when running downhill can be moderated by increasing knee flexion at initial contact and reducing stride length(42). This shock attenuation strategy is also 
associated with a less pronounced heel strike and a forward leaning trunk. Altogether, these protective mechanical adaptations are likely to be specific to MUM and to differentially impact the quadriceps muscles, inducing less stimulation of the RF compared to the vastus muscles, which is clearly illustrated here.

From a mechanistic point of view, $\mathrm{T} 2$ and $\mathrm{T} 2 * \mathrm{MR}$ relaxation measures are well established and sensitive markers of exercise response. For instance, Le Rumeur et al.(32) have shown that $\mathrm{T} 2$ relaxation times can detect different effects of dynamic exercise in trained and untrained subjects and that there was a correlation between $\mathrm{T} 2$ increase and work intensity in healthy volunteers. Beyond the observed mean variations already discussed, individual variations observed in $\mathrm{T} 2$ and $\mathrm{T} 2 *$ values (Figure 4 for $\mathrm{T} 2 *$ and Figure 5 for T2) along such long-distance running are also probably determined by a complex system of regulatory mechanisms including individual response to inflammatory processes at each compartment level, and the intrinsic differences between $\mathrm{T} 2$ and $\mathrm{T} 2 *$ mechanisms can probably provide further insights. Indeed, $\mathrm{T} 2 *$ mapping differentiates from $\mathrm{T} 2$ mapping by its ability to characterize the relaxation of the transverse magnetization that is influenced by macroscopic (inhomogeneities of the magnetic field) and mesoscopic (structure of the tissue) magnetic field inhomogeneities(43-47). For example, reduced $\mathrm{T} 2 *$ values have already shown the potential to describe structural alterations suggestive of ischemic alterations, collagen areas or hemorrhage, for instance, in myocardium 402 and extracardiac tissues $(48,49)$. According to this, histopathologic substrates of $\mathrm{T} 2 *$ and a 403 correlation of $\mathrm{T} 2 *$ to flow analyses have been demonstrated(44-47,50). Recently, reduced T2* 404 values have been described in a group of hypertrophic cardiomyopathy patients potentially triggered through relative ischemia(51). In our case, explanation for the observed increased $\mathrm{T} 2 *$ values could be related to increased perfusion and/or local variation of oxymyoglobin and oxyhemoglobin as oxygen suppliers are increased while deoxymyoglobin is decreased. Note 
408 also that increased $\mathrm{T} 2 *$ contrast will be associated with oxygenated proteins, while 409 deoxygenated proteins that are paramagnetic would reduce local T2* values.

410 Contrary to $\mathrm{T} 2 *, \mathrm{~T} 2$ in muscle is known to have three components: $\sim 18 \%$ of the signal had a 411 very short $\mathrm{T} 2(10 \mathrm{~ms}), 67 \%$ of the signal had an intermediate $\mathrm{T} 2(\sim 40 \mathrm{~ms})$, and the remaining $41215 \%$ of the signal had a long T2 ( 170 ms)(52). The short T2 component is the water bound to 413 macromolecules, and the intermediate and long components represent intracellular and

414 interstitial water, respectively. The literature also clearly describes a unidirectional flow of 415 water from the "innermost" to "outermost" of three compartments connected in series 416 (intracellularly bound water $->$ free intracellular water $\rightarrow$ interstitial water $\rightarrow$ lymphatic 417 vessels). The latter three are subject to considerable variations as a result of normal 418 physiological changes in the muscle. In our study and due to MR acquisition technical 419 constraints, the relaxation in the tissue could be modeled only as a unique compartment. Using 420 a single exponential to describe T2 decay in muscle limits our understanding of the exact 421 contributions of different physiological and biochemical responses to exercise.

\section{PDFF and muscular lipid storage}

424 Our study analyzed noninvasively the local variations in fat content in skeletal muscles during an ultra-endurance running event. With PDFF mapping, MRI is indeed the only noninvasive method that can enable simultaneous, reliable, and repeatable fat fraction estimates within each

427 muscle's heads not achievable by alternative methods such as MR spectroscopy(53).

428 Overall, muscular fat content showed a small decrease at the end of the race, but the changes were not statistically significant except in the most solicited muscle heads (vastus group) (see 430 Figure 3). 
431 Exploring muscular fat content variations during an MUM was of special interest since lipids

432 are the main source of energy in low- to moderate-intensity exercises, such as long-distance

433 events. Additionally, the highest rates of fat oxidation are observed in endurance athletes below

$43465 \%(54,55)$, which is in accordance with what has been measured on the Tor des Géants MUM

435 by Maufrais et al., who reported an average exercise intensity below $50 \%$ of $\mathrm{VO} 2_{\max }(56)$.

436 Muscle fat content is mainly represented by small quantities of triglycerides (TG) that are

437 present as lipid droplets located between or inside muscle fibers (intramyocellular TG, or

438 IMTG), while most fat storage is mainly located in subcutaneous and deep visceral adipose

439 tissue(57). There is now clear evidence that the IMTG pool acts as a buffer regulating fatty

440 acid (FA) flux in skeletal muscle both during exercise to maintain intracellular FA (metabolite)

441 concentrations and in conditions of increased plasma FA provision(58,59). Our findings are in

442 line with other studies that also reported no or minimal reduction of IMTG using biopsies

443 before and after exercise(60-62). Using 1H-MR spectroscopy, other authors reported larger

444 changes with an $25 \%$ IMTG decrease after $2-3 \mathrm{~h}$ of exhaustive running at 50-65\%

$445 \mathrm{VO} 2_{\max }(63,64)$. Both approaches have been challenged as suffering from intrinsic bias: the

446 direct biopsy approach is subject to between-biopsy variability (reported up to $23 \%$ ), while $1 \mathrm{H}$ -

447 MR spectroscopy modeling accuracy may be biased by water content changes and assumptions

448 of FA compositions in IMTG(65). All existing studies have explored fat content changes in

449 short-duration (up to $3 \mathrm{~h}$ ) and high-intensity (up to $80 \% \mathrm{VO}_{2} \mathrm{max}$ ) exercises that profoundly

450 differ from MUM, which intrinsically limits comparison.

451 While the IMTG pool appeared to behave as a buffer to facilitate mitochondrial oxidation and

452 skeletal muscle energy supply, our blood lipid profiles showed a marked reduction in

453 circulating TG and low-density lipoprotein (LDL), since the lipoprotein lipase released by

454 endothelial cells during exercise has been shown to increase FA availability(66). At the same

455 time, we observed an increase in high-density lipoprotein (HDL) cholesterol that coincides 
with significant increases in lipoprotein lipase activity(67) as already reported in long-distance exercise(68).

\section{Paramagnetic susceptibility $\chi$}

Magnetic susceptibility $\chi$ is a physical property that describes the response of a medium being placed within a magnetic field(69). A positive susceptibility value characterizes a paramagnetic medium (O2, salts) with a magnetic field increase, while most biological tissues, including water and fat, are diamagnetic with negative values inducing a local decrease of the initial magnetic field.

All magnetic susceptibility measured in our subjects and all time points were paramagnetic coherently with the metabolic consumption of oxygen in tissues: the weakly diamagnetic oxyhemoglobin releases strongly paramagnetic $\mathrm{O} 2$ and deoxyhemoglobin molecules(70). The magnetic susceptibility $\chi$ remained very stable with only a minimal increase at arrival of $1.75 \pm 0.43$ ppm vs. $1.49 \pm 0.34$ ppm at baseline (see Supplemental Digital Content 8 , Values of urinary and blood biomarkers at 4 time points), as expected in low-intensity aerobic exercise where the average intensity remains below $50 \%$ of $\mathrm{VO} 2 \max (56)$, explaining the lack of significant variations over time. Indeed, measurable changes in magnetic susceptibility-related measures in skeletal muscles reported in the literature occurred only in critical conditions such as cuff compression tests or ischemia(71). Our differences among muscle heads and groups are, however, in line with findings by others(71). This finding is explained by the differences among muscles in slow-twitch oxidative muscle fibers, different capillary density, and myoglobin content.

From a broader perspective, the proposed approach could also serve in diseases for longitudinal monitoring of muscular disorder progression or improvement due to various treatments. 
480

481

482

483

484

485

486

487

488

489

490

\section{$491 \quad$ Limitations}

492 While we have studied the average response for all the subjects, there is an interindividual 493 response heterogeneity which is likely influenced by factors such as age(77), training 494 status(78), performance level, experience, and many others that likely modulated the 495

Indeed, the motivation for both athletes and physicians during this study was also to serve and encourage technological developments that could, beyond a deeper understanding of muscle physiology during extreme exercise, also help, in turn, be a crucial adjunct to improve patient diagnosis, follow-up, and care, evaluate the effects of personalized-designed therapies, a highly critical objective not in clinical routine. Indeed, there are several clinical scenarios in which pathologies lead to inflammation or muscle wasting. Moreover, such an approach could be coupled to the other strengths of MRI, including in vivo dynamic imaging of muscle function and kinematics(72-75), muscle microstructure using diffusion MRI or measures of chemical changes associated with metabolic states and glycogen consumption using MR $\operatorname{spectroscopy}(76)$.

\footnotetext{
inflammatory response but could not be taken into account due to our limited sample size.
}

In this paper, we used simple statistical descriptors to analyze the quantitative indexes extracted at each time point and within each muscle head, while radiomic features could have been explored for each quantitative index.

While considering the results on PDFF, it should be kept in mind that the PDFF values in our study are especially low since our subjects are professional athletes and there are estimation errors in PDFF map computation. 
502 Recently, more specific acquisition methods and modeling, combining ultrashort-TE 503 acquisition(79) and muscle-specific models of diffusion, might enable short-term

504 distinguishing the $\mathrm{T} 2$ of each muscle compartment (T2 intracellular, $\mathrm{T} 2$ in the membrane, $\mathrm{T} 2$

505 extracellular) and thus elucidating the different causes of changes in the parameters.

\section{Conclusion}

507 Using a model of prolonged and extreme mechanical stress, we showed that a dedicated 508 analysis pipeline enables the extraction of local quantitative imaging markers of inflammation 509 and metabolic response and the detection of local changes related to exercise. Our study 510 confirms that prolonged MUMs induce singular muscle damages compared to other sporting 511 events, while the extreme eccentric load (due to the $24000 \mathrm{~m}$ of downhill running) is likely the 512 main trigger. When applied with proposed segmentation and analysis pipeline, qMRI can 513 monitor these changes while differentiating the involvement of the heads of the quadriceps 514 muscle.

515 There are many potential applications; for example, in sports science, our approach could be 516 used to explore the impact of other exercise challenges or to quantify the effects of training 517 strategies or training programs on specific individuals. Considering that MRI can also provide 518 high-spatial-resolution anatomical images of muscle injuries and trauma, as well as information 519 on muscle microstructure and metabolic variables such as glycogen consumption, qMRI would 520 be a highly relevant noninvasive modality for investigating the relationship among force loss, 521 muscle microstructure, performance and recovery in athletes as well as muscle wasting and 522 disability in patients. 


\section{ACKNOWLEDGEMENTS}

524 This work has been performed within the framework of LABEX PRIMES (ANR-11-LABX-

525 0063) of Université de Lyon, within the program "Investissements d'Avenir" (ANR-11-IDEX-

526 0007) and France Life Imaging (ANR-11-INBS-0006) operated by the French National

527 Research Agency (ANR). H-T.Nguyen is working under doctoral contract with 488 SIS

528 doctoral school of Université de Lyon. The authors also thank warmly all ultra-trailers that

529 volunteered for the MUST project, Dr Guido Girardini from the Valle d'Aosta Regional for his

530 unfailing support and help, Hospital the Aosta Valley Regional Government, the VDA trailers

531 and Courmayeur city representatives for their technical assistance. 


\section{CONFLICT OF INTEREST}

533 The authors declare that there is no conflict of interest. The results of the present study do not

534 constitute endorsement by ACSM and are presented clearly, honestly, and without fabrication,

535 falsification, or inappropriate data manipulation. 


\section{REFERENCES}

537 1. Millet GP, Millet GY. Ultramarathon is an outstanding model for the study of adaptive responses to extreme load and stress. BMC Med [Internet]. 2012;10(1):1-3. Available from: http://dx.doi.org/10.1186/1741-7015-10-77

2. Andonian P, Viallon M, Le Goff C, et al. Shear-wave elastography assessments of quadriceps stiffness changes prior to, during and after prolonged exercise: A longitudinal study during an extreme mountain ultra-marathon. PLoS One. 2016;11(11):1-21.

544

3. Zanchi D, Viallon M, Goff C Le, et al. Extreme mountain ultra-marathon leads to acute but transient increase in cerebral water diffusivity and plasma biomarkers levels changes. Front Physiol. 2017;7(JAN).

547 4. Belli T, Macedo DV, De Araújo GG, et al. Mountain ultramarathon induces early increases of muscle damage, inflammation, and risk for acute renal injury. Front Physiol. 2018;9:1368.

5. Saugy J, Place N, Millet GY, Degache F, Schena F, Millet GP. Alterations of Neuromuscular Function after the World's Most Challenging Mountain UltraMarathon. PLoS One [Internet]. 2013;8(6):1-11. Available from: http://dx.doi.org/10.1371\%2Fjournal.pone.0065596

554 6. Clarkson PM, Hubal MJ. Exercise-induced muscle damage in humans. Am J Phys Med Rehabil. 2002;81(11 Supp):S52-69.

556 7. Guilhem G, Cornu C, Maffiuletti NA, Guével A. Neuromuscular adaptations to isoload versus isokinetic eccentric resistance training. Med Sci Sports Exerc. 2013;45(2):32635.

559 8. Brown RW, Cheng YCN, Haacke EM, Thompson MR, Venkatesan R. Magnetic 
561 9. Saab G, Thompson RT, Marsh GD. Multicomponent T2 relaxation of in vivo skeletal muscle. Magn Reson Med. 1999;42:150-7.

563 10. Ababneh ZQ, Ababneh R, Maier SE, et al. On the correlation between T2 and tissue diffusion coefficients in exercised muscle: Quantitative measurements at 3T within the tibialis anterior. Magn Reson Mater Physics, Biol Med. 2008;21:273-8.

11. Ploutz-Snyder LL, Nyren S, Cooper TG, Potchen EJ, Meyer RA. Different effects of exercise and edema on T2 relaxation in skeletal muscle. Magn Reson Med. $1997 ; 37: 676-82$.

12. Ran J, Ji S, Morelli JN, Wu G, Li X. T2 mapping in dermatomyositis/polymyositis and correlation with clinical parameters. Clin Radiol. 2018;73(12):1057.e13-1057.e8.

13. Maillard SM, Jones R, Owens C, et al. Quantitative assessment of MRI T2 relaxation time of thigh muscles in juvenile dermatomyositis. Rheumatology. 2004;43(5):603-8.

573 14. Thuny F, Lairez O, Roubille F, et al. Post-conditioning reduces infarct size and edema in patients with ST-segment elevation myocardial infarction. J Am Coll Cardiol. 2012;59(24):2175-81.

15. Biglands JD, Grainger AJ, Robinson P, et al. MRI in acute muscle tears in athletes: can quantitative T2 and DTI predict return to play better than visual assessment? Eur Radiol. 2020;

579 16. Welsch GH, Hennig FF, Krinner S, Trattnig S. T2 and T2* Mapping. Current Radiology Reports. 2014. p. 60.

581 17. Froeling M, Oudeman J, Strijkers GJ, et al. Muscle Changes Detected with DiffusionTensor Imaging after Long-Distance Running. Radiology [Internet]. 2015;274(2):548- 
18. Maeo S, Ando Y, Kanehisa H, Kawakami Y. Localization of damage in the human leg muscles induced by downhill running. Sci Rep. 2017;7(1):5769.

19. Leporq B, Le Troter A, Le Fur Y, et al. Combined quantification of fatty infiltration, T 1-relaxation times and $\mathrm{T} 2 *$-relaxation times in normal-appearing skeletal muscle of controls and dystrophic patients. Magn Reson Mater Physics, Biol Med [Internet]. 2017 Aug;30(4):407-15. Available from: https://doi.org/10.1007/s10334-017-0616-1

20. Leporq B, Ratiney H, Pilleul F, Beuf O. Liver fat volume fraction quantification with fat and water T1 and T2*estimation and accounting for NMR multiple components in patients with chronic liver disease at 1.5 and 3.0 T. Eur Radiol. 2013;23(8):2175-86.

21. Tawara N, Nitta O, Kuruma H, Niitsu M, Itoh A. T2 mapping of muscle activity using ultrafast imaging. Magn Reson Med Sci. 2011;10(2):85-91.

22. Patten C, Meyer RA, Fleckenstein JL. T2 mapping of muscle. Semin Musculoskelet Radiol. 2003;7(4):297-305.

23. Gilles B, De Bourguignon C, Croisille P, Millet G, Beuf O, Viallon M. Automatic 598 segmentation for volume quantification of quadriceps muscle head: a longitudinal study in athletes enrolled in extreme mountain ultra-marathon. In: ISMRM2016: International Society for Magnetic Resonance in Medicine. Singapore;

24. Bakdash JZ, Marusich LR. Repeated measures correlation. Front Psychol. 2017;42(2):261-7.

25. Fernandez NF, Gundersen GW, Rahman A, et al. Clustergrammer, a web-based heatmap visualization and analysis tool for high-dimensional biological data. Sci Data. 2017;4:170151. 
26. Lucas SJE, Helge JW, Schütz UHW, Goldman RF, Cotter JD. Moving in extreme environments: Extreme loading; carriage versus distance. Extrem Physiol Med. 2016;5:6.

27. Schütz UHW, Billich C, König K, et al. Characteristics, changes and influence of body composition during a $4486 \mathrm{~km}$ transcontinental ultramarathon: Results from the Transeurope Footrace mobile whole body MRI-project. BMC Med. 2013;11(1):122.

28. Theysohn JM, Kraff O, Maderwald S, et al. MRI of the ankle joint in healthy nonathletes and in marathon runners: Image quality issues at 7.0 $\mathrm{T}$ compared to $1.5 \mathrm{~T}$. Skeletal Radiol. 2013;42(2):261-7.

29. Fulford J, Eston RG, Rowlands A V, Davies RC. Assessment of magnetic resonance techniques to measure muscle damage $24 \mathrm{~h}$ after eccentric exercise. Scand J Med Sci Sport. 2014;25(1):e28-39.

30. Fleckenstein JL. Muscle water shifts, volume changes, and proton T2 relaxation times after exercise. J Appl Physiol. 1993;74(4):2047-8.

31. Fleckenstein JL, Watumull D, McIntire DD, Bertocci LA, Chason DP, Peshock RM. Muscle proton $\mathrm{T} 2$ relaxation times and work during repetitive maximal voluntary exercise. J Appl Physiol. 1993;74(6):2855-9.

32. Le Rumeur E, Carre F, Bernard AM, Bansard JY, Rochcongar P, De Certaines JD. Multiparametric classification of muscle $\mathrm{T} 1$ and $\mathrm{T} 2$ relaxation times determined by magnetic resonance imaging. The effects of dynamic exercise in trained and untrained subjects. Br J Radiol. 1994;67(794):150-6.

33. Skenderi KP, Kavouras SA, Anastasiou CA, Yiannakouris N, Matalas AL. Exertional rhabdomyolysis during a 246-km continuous running race. Med Sci Sports Exerc. 2006;38(6):1054-7. 
630 34. Fridén J, Lieber RL. Eccentric exercise-induced injuries to contractile and cytoskeletal muscle fibre components. Acta Physiol Scand. 2001;171(2):321-6.

35. Robach P, Boisson RC, Vincent L, et al. Hemolysis induced by an extreme mountain ultra-marathon is not associated with a decrease in total red blood cell volume. Scand J Med Sci Sport. 2014;24(1):18-27.

36. Overgaard K, Lindstrom T, Ingemann-Hansen T, Clausen T. Membrane leakage and increased content of $\mathrm{Na}+\mathrm{K}+$ pumps and $\mathrm{Ca} 2+$ in human muscle after a $100-\mathrm{km}$ run. $\mathrm{J}$ Appl Physiol. 2002;92(5):1891-8.

37. McCarthy DA, Dale MM. The Leucocytosis of Exercise: A Review and Model. Sport Med. 1988;6(6):333-63.

38. Hikida RS, Staron RS, Hagerman FC, Sherman WM, Costill DL. Muscle fiber necrosis associated with human marathon runners. J Neurol Sci. 1983;59(2):185-203.

39. Knechtle B, Duff B, Schulze I, Kohler G. A multi-stage ultra-endurance run over 1,200 $\mathrm{km}$ leads to a continuous accumulation of total body water. J Sport Sci Med. 2008;7(3):357-64.

40. Sloniger MA, Cureton KJ, Prior BM, Evans EM. Lower extremity muscle activation during horizontal and uphill running. J Appl Physiol [Internet]. 1997;83(6):2073-9. Available from: http://jap.physiology.org/content/83/6/2073

41. Degache F, Morin JB, Oehen L, et al. Running mechanics during the world's most challenging mountain ultramarathon. Int J Sports Physiol Perform. 2016;11(5):608-14.

42. Gottschall JS, Kram R. Ground reaction forces during downhill and uphill running. J Biomech. 2005;38(3):445-52.

652 43. Van Oorschot JWM, Gho JMIH, Van Hout GPJ, et al. Endogenous contrast MRI of 
cardiac fibrosis: Beyond late gadolinium enhancement. J Magn Reson Imaging. 2015;41(5):1181-9.

44. Van Nierop BJ, Bax NAM, Nelissen JL, et al. Assessment of myocardial fibrosis in mice using a T2*-weighted 3D radial magnetic resonance imaging sequence. PLoS One. 2015;10(6):e0129899.

45. Huang S yun, Li X hua, Huang L, et al. T2* Mapping to characterize intestinal fibrosis in crohn's disease. J Magn Reson Imaging. 2018;48(3).

46. Zia MI, Ghugre NR, Connelly KA, et al. Characterizing myocardial edema and hemorrhage using quantitative $\mathrm{t} 2$ and $\mathrm{t} 2 *$ mapping at multiple time intervals post STsegment elevation myocardial infarction. Circ Cardiovasc Imaging. 2012;5(5):566-72.

47. Messroghli DR, Moon JC, Ferreira VM, et al. Clinical recommendations for cardiovascular magnetic resonance mapping of $\mathrm{T} 1, \mathrm{~T} 2, \mathrm{~T} 2$ and extracellular volume: A consensus statement by the Society for Cardiovascular Magnetic Resonance (SCMR) endorsed by the European Association for Cardiovascular Imagin. J Cardiovasc Magn Reson. 2017;19(1):75.

48. Manka R, Paetsch I, Schnackenburg B, Gebker R, Fleck E, Jahnke C. BOLD cardiovascular magnetic resonance at 3.0 tesla in myocardial ischemia. J Cardiovasc Magn Reson. 2010;12(1):54.

49. Jahnke C, Gebker R, Manka R, Schnackenburg B, Fleck E, Paetsch I. Navigator-gated 3D blood oxygen leveldependent CMR at 3.0-T for detection of stress-induced myocardial ischemic reactions. JACC Cardiovasc Imaging. 2010;3(4):375-84.

50. Aguor ENE, Arslan F, Van De Kolk CWA, et al. Quantitative T2 * assessment of acute and chronic myocardial ischemia/reperfusion injury in mice. Magn Reson Mater Physics, Biol Med. 2012;25(5):369-79. 
677 51. Lira FS, Yamashita AS, Uchida MC, et al. Low and moderate, rather than high

678

679

680

681

682

683

684

685

686

687

688

689

690

691

692

693

694

695

696

697

698

699

700 intensity strength exercise induces benefit regarding plasma lipid profile. Diabetol Metab Syndr. 2010;3:31.

52. Belton PS, Jackson RR, Packer KJ. Pulsed NMR studies of water in striated muscle. I. Transverse nuclear spin relaxation times and freezing effects. BBA - Gen Subj. 1972;286(1):16-25.

53. Nemeth A, Segrestin B, Leporq B, et al. Comparison of MRI-derived vs. traditional estimations of fatty acid composition from MR spectroscopy signals. NMR Biomed. 2018;31(9):e3991.

54. Achten J, Gleeson M, Jeukendrup AE. Determination of the exercise intensity that elicits maximal fat oxidation. Med Sci Sports Exerc. 2002;42(4):405-12.

55. Jeukendrup AE, Saris WHM, Wagenmakers AJM. Fat metabolism during exercise: A review - Part II: Regulation of metabolism and the effects of training. Int J Sports Med. 1998;19(5):203-302.

56. Maufrais C, Millet GP, Schuster I, Rupp T, Nottin S. Progressive and biphasic cardiac responses during extreme mountain ultramarathon. Am J Physiol - Hear Circ Physiol [Internet]. 2016;310(10):H1340-8. Available from: http://ajpheart.physiology.org/content/310/10/H1340

57. Spriet LL. Regulation of skeletal muscle fat oxidation during exercise in humans. Med Sci Sports Exerc. 2002;34(9):1477-84.

58. Watt MJ, Cheng Y. Triglyceride metabolism in exercising muscle. Biochim Biophys Acta - Mol Cell Biol Lipids. 2017;1862(10 Pt B):1250-9.

59. Van Loon LJC. Use of intramuscular triacylglycerol as a substrate source during exercise in humans. J Appl Physiol. 2004;97(4):1170-87. 
701

702

703

704

705

706

707

708

709

710

711

712

713

714

715

716

717

718

719

720

721

722

723

724

60. Stankiewicz-Choroszucha B, Górski J. Effect of decreased availability of substrates on intramuscular triglyceride utilization during exercise. Eur J Appl Physiol Occup Physiol. 1978;40(1):27-35.

61. Kiens B, Essen-Gustavsson B, Christensen NJ, Saltin B. Skeletal muscle substrate utilization during submaximal exercise in man: effect of endurance training. J Physiol. 1993;469(459-78).

62. Guo Z, Burguera B, Jensen MD. Kinetics of intramuscular triglyceride fatty acids in exercising humans. J Appl Physiol. 2000;89(5):2057-64.

63. Krssak M, Petersen KF, Bergeron R, et al. Intramuscular glycogen and intramyocellular lipid utilization during prolonged exercise and recovery in man: A 13C and 1H nuclear magnetic resonance spectroscopy study. J Clin Endocrinol Metab. $2000 ; 85(2): 748-54$.

64. Décombaz J, Schmitt B, Ith M, et al. Postexercise fat intake repletes intramyocellular lipids but no faster in trained than in sedentary subjects. Am J Physiol - Regul Integr Comp Physiol. 2001;281(3):R760-9.

65. Watt MJ, Heigenhauser GJF, Spriet LL. Intramuscular triacylglycerol utilization in human skeletal muscle during exercise: Is there a controversy? J Appl Physiol. 2002;93(4):1185-95.

66. Kiens B, Roepstorff C, Glatz JFC, et al. Lipid-binding proteins and lipoprotein lipase activity in human skeletal muscle: Influence of physical activity and gender. J Appl Physiol. 2004;97(4):1209-18.

67. Ferguson M a, Alderson NL, Trost SG, Essig D a, Burke JR, Durstine JL. Effects of four different single exercise sessions on lipids, lipoproteins, and lipoprotein lipase. J Appl Physiol. 1998;85(3):1169-74. 
725 68. Frias MA, Virzi J, Golaz O, Gencer B, Mach F, Vuilleumier N. Impact of long distance rowing on biological health: A pilot study. Clin Biochem. 2018;52(142-147).

727

728

729

730

731

732

733

734

735

736 737

738

739

740

741

742

743

744

745

746

747

69. Schenck JF. The role of magnetic susceptibility in magnetic resonance imaging: MRI magnetic compatibility of the first and second kinds. Med Phys. 1996;23(6):815-50.

70. Wang Y, Liu T. Quantitative susceptibility mapping (QSM): Decoding MRI data for a tissue magnetic biomarker. Magn Reson Med. 2015;73(1):82-101.

71. Wang C, Zhang R, Zhang X, et al. Noninvasive measurement of lower extremity muscle oxygen extraction fraction under cuff compression paradigm. J Magn Reson Imaging. 2016;43(5):1148-58.

72. Drace JE, Pelc NJ. Measurement of skeletal muscle motion in vivo with phase-contrast MR imaging. J Magn Reson Imaging. 1994;4(2):157-63.

73. Pappas GP, Asakawa DS, Delp SL, Zajac FE, Drace JE. Nonuniform shortening in the biceps brachii during elbow flexion. J Appl Physiol. 2002;92(6):2381-9.

74. Asakawa DS, Blemker SS, Gold GE, Delp SL. In vivo motion of the rectus femoris muscle after tendon transfer surgery. J Biomech. 2002;35(8):1029-37.

75. Zhong X, Epstein FH, Spottiswoode BS, Helm PA, Blemker SS. Imaging twodimensional displacements and strains in skeletal muscle during joint motion by cine DENSE MR. J Biomech. 2008;41(3):532-40.

76. Kent-Braun J a, McCully KK, Chance B. Metabolic effects of training in humans: a 31P-MRS study. J Appl Physiol. 1990;69(3):1165-70.

77. Manfredi TG, Fielding RA, O’Reilly KP, Meredith CN, Lee HY, Evans WJ. Plasma creatine kinase activity and exercise-induced muscle damage in older men. Med Sci Sports Exerc. 1991;23(9):1028-34. 
748 78. Newton MJ, Morgan GT, Sacco P, Chapman DW, Nosaka K. Comparison of Responses to Strenuous Eccentric Exercise of the Elbow Flexors Between ResistanceTrained and Untrained Men. J Strength Cond Res. 2008;22(2):597-607.

751 79. Saab G, Thompson RT, Marsh GD. Multicomponent T2 relaxation of in vivo skeletal 752 muscle. Magn Reson Med. 1999;42(1):150-7.

753 80. Kikinis R, Pieper SD, Vosburgh KG. 3D Slicer: A Platform for Subject-Specific Image 754 Analysis, Visualization, and Clinical Support. In: Jolesz FA, editor. Intraoperative 755 Imaging and Image-Guided Therapy [Internet]. New York, NY: Springer New York; 2014. p. 277-89. Available from: https://doi.org/10.1007/978-1-4614-7657-3_19 
760 Figure 1: Flowchart summarizing the main steps of our study. MRI was performed at three 761 time points: before (Pre), and participants who finished the race, immediately after (Post) and $762 \sim 48-72 \mathrm{~h}$ after the race (Post+3). Meanwhile, biological sampling was performed at four time 763 points (an additional sample was performed at half-race (Mid)).

764 Figure 2: MRI input sequences superposed on the isotropic water image (a), the coronal view 765 of the automatic segmentation displayed with the isotropic water image as a background (b) 766 and its 3D volume (c) rendered with 3DSlicer(80).

767 Figure 3: t-test matrix with color-coded P-values for multiple comparisons of qMRI metrics $768(\chi$, PDFF, T2, T2*) between muscle heads at all three acquisition time points (Pre, Post, 769 Post+3). Abbreviations: VL - Vastus Lateralis, RF - Rectus Femoris, VM - Vastus Medialis, 770 VI - Vastus Intermedius, $r$ - right, 1 - left. For easy viewing, dotted diagonals highlight inter771 leg (right/left) comparisons of the same muscle heads at each time point, while all other boxes 772 are intra-leg and/or inter-head comparisons.

773 Figure 4: Variation of T2* mean in the individual muscle heads of all finishers with an example 774 of $\mathrm{T} 2 *$ maps at the three MR acquisition time points relative to the race of the same subject. A 775 P-value less than .05 indicates a significant change between two time points. Abbreviations: 776 VL - Vastus Lateralis, RF - Rectus Femoris, VM - Vastus Medialis, VI - Vastus Intermedius.

777 Figure 5: Variation of T2 mean in the individual muscle heads of all finishers with an example 778 of T2 maps at the three MR acquisition time points relative to the race of the same subject. A 779 P-value less than .05 indicates a significant change between two time points. Abbreviations: 780 VL - Vastus Lateralis, RF - Rectus Femoris, VM - Vastus Medialis, VI - Vastus Intermedius.

781 Figure 6: Variation of PDFF mean in the individual muscle heads of all finishers with an 782 example of PDFF maps at the three acquisition time points relative to the race of the same 
783 subject. A P-value less than .05 indicates significant changes between two time points. 784 Abbreviations: VL - Vastus Lateralis, RF - Rectus Femoris, VM - Vastus Medialis, VI 785 Vastus Intermedius.

786 Figure 7: Variation of $\chi$ mean in the individual muscle heads of all finishers with an example 787 of $\chi$ maps at the three acquisition time points relative to the race of the same subject. A P-value 788 less than .05 indicates a significant change between two time points. Abbreviations: VL 789 Vastus Lateralis, RF - Rectus Femoris, VM - Vastus Medialis, VI - Vastus Intermedius.

790 Figure 8: P-values of statistical tests in our longitudinal analysis on the blood and urinary 791 biomarker datasets. A P-value less than .05 indicates a significant change between two time 792 points. 


\section{Supplemental Digital Contents}

794 SDC1.docx MR imaging acquisition parameters

795 SDC2.docx Quantitative maps computation

796 SDC3.docx Muscle segmentation

797 SDC4.docx List of 58 biological markers

798 SDC5.docx Biological data preprocessing for missing data

799 SDC6.docx Demographic data and qMRI metrics of the subject population

800 SDC7.docx Specific longitudinal T2* analysis

801 SDC8.docx Values of urinary and blood biomarkers at 4 time points

802 SDC9.docx Correlation between biological markers and image features extracted from 803 quadriceps muscles in each qMRI quantitative map 